\title{
The role of the self-consistent equation in identifying the Andreev spectrum in a mesoscopic NS structure
}

\author{
G.A. Gogadze and S.N. Dolya \\ B. Verkin Institute for Low Temperature Physics and Engineering of the National Academy of Sciences of Ukraine \\ 47 Lenin Ave., Kharkov 61103, Ukraine \\ E-mail: GOGADZE@ilt.kharkov.ua
}

Received March 31, 2009

\begin{abstract}
A differential self-consistent equation has been obtained for a dimensionless magnetic flux in a NS structure, which is responsible for the magnetic moment jumps in the system.
\end{abstract}

PACS: 74.45.+c Proximity effects; Andreev effect; SN and SNS junctions;

74.50.+r Tunneling phenomena; point contacts, weak links, Josephson effects.

Keywords: magnetic susceptibility, normal metal-superconductor structures, coherent phenomena.

The law of conduction electron dispersion manifests itself in all properties of the metal. The knowledge of the spectrum enables one to calculate the thermodynamic properties of metallic samples. B.I. Verkin has contributed much to investigations of a quasiparticle spectrum in normal metals. He supported and promoted research in physics of coherent phenomena in normal metals and superconductors.

The coherent phenomena modified by the proximity effect in cylindrical mesoscopic normal metal (N)-superconductor (S) structures have been considered. Such structures (superconducting $\mathrm{Nb}$ wires with radii $R$ of tens of microns covered with a thin layer $d$ of a pure normal metal $\mathrm{Ag}, \mathrm{Au}$ or $\mathrm{Cu}$ ) were investigated experimentally by Mota et al. [1-3]. The magnetic susceptibility of these NS structures [1-3] exhibited an anomalous behavior in weak magnetic fields at millikelvin temperatures. The phenomenon was called a reentrant effect. For a long time no adequate theory was proposed to interpret it. Its origin have been explained in Refs. 4-6. The theory of the reentrant effect is essentially based on the properties of the Andreev levels [7] in a NS structure experiencing a magnetic field. The fields are taken to be so weak that the bending of quasiparticle paths is negligible. The role of the field reduces to displaying the Aharonov-Bohm effect [8], i.e., to accounting for the incursion in of the wave function phase of a quasiparticle on its movement along the trajectory in the vector potential field. The spectrum of quasiparticles was taken in Refs. 4, 5 using the method of multidimensional quasiclassics $[9,10]$. It has the form [5]

$$
\begin{gathered}
\mathrm{E}_{n}(q, \alpha, \Phi)=\frac{\pi \hbar v_{L}(q) \cos \alpha}{2 d} \times \\
\times\left[n+\frac{1}{\pi} \arccos \left(\frac{\mathrm{E}_{n}(q, \alpha, \Phi)}{\Delta}\right)-\frac{\tan \alpha}{\pi} \Phi\right] .
\end{gathered}
$$

Here $v_{L}(q)=\sqrt{p_{F}^{2}-q^{2}} / m^{*}, q$ is the component of the quasimomentum along the symmetry axis of the cylinder, $m^{*}$ is the effective mass of a quasiparticle, $d$ is the N-layer thickness, $\alpha$ is the angle of the quasiparticle dielectric boundary collision, $\Delta$ is the energy gap. The $\Phi$ in the last term of Eq. (1) has the meaning of a «phase»

$$
\Phi=\frac{2 \pi}{\Phi_{0}} \int_{0}^{d} A(x) d x .
$$

Note that the quasiparticle spectrum in Eq. (1) cannot be identified unambiguously unless the dependence of the «phase» $\Phi$ on the magnetic field is calculated. This fact is of fundamental importance for understanding the physics of the reentrant effect.

The spectrum in Eq. (1) features an important property: when the «phase» $\Phi$ changes, the density of states

$$
v(\varepsilon, \Phi)=\sum_{n, \alpha, \sigma} \int \delta\left(\varepsilon-E_{n}(q, \alpha, \Phi)\right) d q
$$


exhibits resonance spikes [4,5]. Every time when the Andreev level coincides with the chemical potential of the metal, the state of the NS structure experiences strong degeneracy seen as a spike.

To calculate the "phase» $\Phi(T, h)$, one should know how the vector potential field is distributed inside the normal metal. It is shown by Zaikin [11] that the distribution of the vector potential field in the $\mathrm{N}$ layer is inhomogeneous. He derived an algebraic self-consistent equation for a dimensionless flux («phase» $\Phi)$

$$
\Phi(T, H)=h+\text { const } \cdot j(\Phi),
$$

where $h=H / H_{0}, H_{0}=\Phi_{0} /\left(\pi d^{2} \mu_{0}\right), \mu_{0}$ is the magnetic permeability in vacuum.

The term $j(\Phi)$ in Eq. (3) is described by Eq. (13) of Ref. 11, which was obtained proceeding from Eilenberger equations [12].

Our calculation of the magnetic moment (persistent current) is based on the thermodynamic interpretation of current as

$$
M(T, H)=-\frac{d \Omega}{d H}=-\frac{d \Omega}{d \Phi} \frac{d \Phi}{d H},
$$

where $\Omega(\Phi)$ is the thermodynamic potential of the structure (the Boltzmann constant is unity):

$$
\Omega(\Phi)=-T \sum_{n, q, \alpha, s} \ln \left[1+\exp \left(-\frac{E_{n}(q, \alpha ; \Phi)}{T}\right)\right],
$$

where summation is over the spin $s$ and all the states relating to the quasiparticle trajectories with $\alpha \leq \alpha_{c}, \alpha_{c}$ is the angle at which the trajectory touches the NS boundary, $\sin \alpha_{c}=R /(R+d)$. The trajectories $\alpha>\alpha_{c}$ do not collide with the NS boundary and do not contribute to the
Andreev spectrum. They are therefore disregarded in further consideration.

Let us introduce dimensionless parameters

$$
\varepsilon_{n}=\frac{E_{n}(q, \alpha, \Phi)}{\Delta}, \quad \sigma=\frac{\hbar p_{F}}{2 d \Delta m^{*}}, \quad-1 \leq \varepsilon_{n} \leq 1,
$$

and perform substitution of the variables $\{q, \alpha\} \rightarrow\{u, v\}$

$$
\left\{\begin{array}{l}
u=\sigma \sqrt{1-\left(\frac{q}{p_{F}}\right)^{2}} \cos \alpha \\
v=\sigma \sqrt{1-\left(\frac{q}{p_{F}}\right)^{2}} \sin \alpha
\end{array} .\right.
$$

The spectrum in Eq. (1) becomes:

$$
\varepsilon_{n}=\left[n \pi+\arccos \varepsilon_{n}\right] u-\Phi v,
$$

where $0 \leq u \leq \sigma,-\sigma \sin \alpha_{c} \leq v \leq \sigma \sin \alpha_{c}$ and the spectrum symmetry $\varepsilon_{-|n|}(u, v, \Phi)=-\varepsilon_{|n|-1}(u,-v, \Phi)$ is taken into account.

Using the derivative

$$
\frac{d \varepsilon_{n}}{d \Phi}=-\frac{v \sqrt{1-\varepsilon_{n}^{2}}}{u+\sqrt{1-\varepsilon_{n}^{2}}},
$$

we can write the expression for the derivative of the thermodynamic potential with respect to the flux $M^{*}(\Phi)=$ $=-d \Omega / d \Phi$. This quantity differs from the magnetic moment

$$
M(H)=-\frac{d \Omega}{d H}=M^{*}(\Phi) \frac{d \Phi}{d H}
$$

in the absence of the factor $d \Phi / d H$ :

$$
M^{*}(\Phi)=c_{3} \sum_{n=0}^{n=\infty} \iint_{R} \frac{u v \tanh \left(\frac{\varepsilon_{n}(u, v, \Phi)}{2 c_{2} T}\right) \sqrt{1-\varepsilon_{n}^{2}(u, v, \Phi)}}{\left(u+\sqrt{1-\varepsilon_{n}^{2}(u, v, \Phi)}\right) \sqrt{\sigma^{2}-u^{2}-v^{2}}} d u d v
$$

where

$$
c_{2}=\frac{k_{B}}{\Delta}, c_{3}=\frac{R \Delta}{\pi}\left(\frac{p_{F}}{\sigma \hbar}\right)^{2}
$$

We obtain a differential equation for $\Phi(T, h)$ instead of Eq. (3):

$$
\Phi(T, h)=h+\eta M^{*}(T, \Phi) \frac{\partial \Phi(T, h)}{\partial h},
$$

where $\eta=d^{2} /\left(3 R^{2} \Phi_{0} H_{0}\right)$. It is of great importance for calculating the magnetic moment (current) of the NS structure.

The dimensionless flux $\Phi$ is depended both on the magnetic field and on temperature. The magnetic moment (screening current) of the NS structure contains the factor $\partial \Phi(T, h) / \partial h$. The condition of self-consistency describes the nonlinear behavior of the flux $\Phi$ as a function of the magnetic field. The magnetic susceptibility is defined as a derivative of the magnetic moment with respect to the 


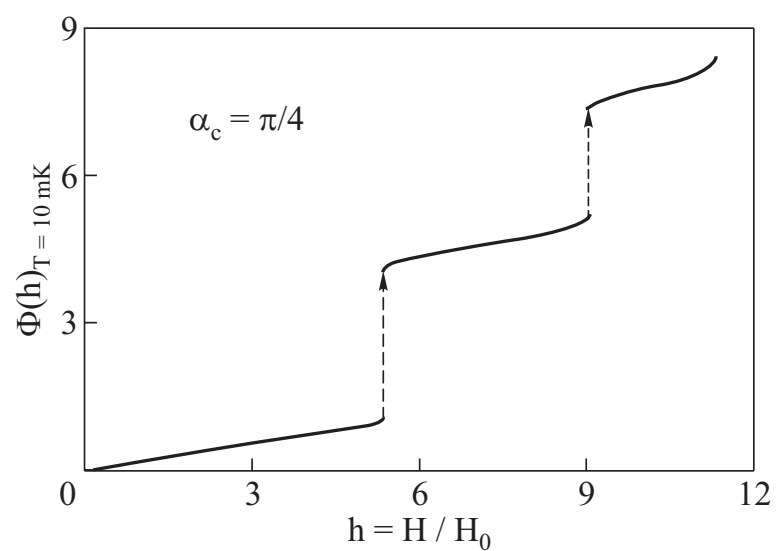

Fig. 1. The dependence $\Phi(T, h)$ as a function of magnetic field $h$.

magnetic field in the limiting case of weak magnetic field. Therefore, in weak fields $\partial \Phi(T, h) / \partial h$ is a constant and the screening current-magnetic flux dependence is linear. However, the nonlinearity effect becomes essential in a growing magnetic field, which leads to unusual dependences of current (magnetic moment) on the magnetic field.

The dependence $\Phi(T, h)$ obtained through a numerical solution of self-consistent Eq. (8) exhibits jumps (see Fig. 1).

Figure 2 illustrates the dependence $M^{*}(\Phi)$ of Eq. (7) calculated numerically for a particular NbAu sample [3]. The sample sizes are $R=8 \mu \mathrm{m}, d=3.3 \mu \mathrm{m}$. The characteristics of the material are $T_{c}(\mathrm{Nb})=9.26 \mathrm{~K}, k_{F}(\mathrm{Au})=$ $=1.2 \cdot 10^{8} \mathrm{~cm} / \mathrm{s}, \alpha=\pi / 4$ (the critical angle of the trajectory), $H_{0}=0.31 \mathrm{Oe}(\approx 51 \mathrm{~A} / \mathrm{m}) ; \eta c_{3}=5 \cdot 10^{3}, \sigma=0.64$ are the dimensionless quantities.

The numerically calculated isothermal dependence of the magnetic moment of the NS structure upon the mag-

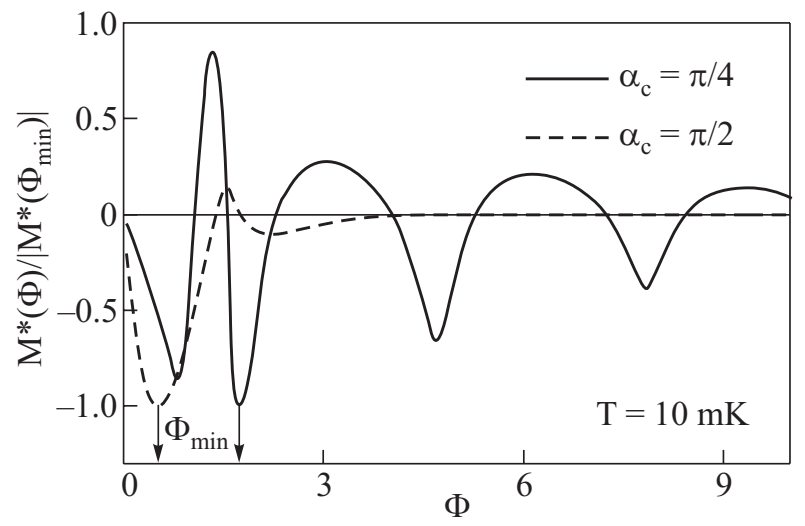

Fig. 2. The ratio $M^{*}(\Phi) / M^{*}\left(\Phi_{\min }\right)($ Eq. (7)) as a function of the flux $\Phi$.

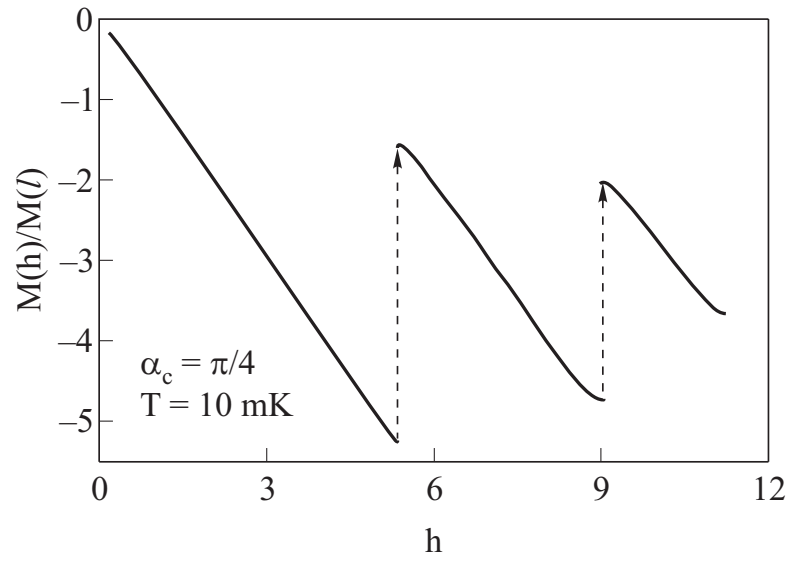

Fig. 3. The behavior of the magnetic moment of the NS structure as a function of the field $h$.

netic field is shown in Fig. 3. It is seen that the magnetic moment of the NS structure is always diamagnetic, but its magnitude changes stepwise with an increasing magnetic field. This behavior can be attributed to the "paramagnetic» additive appearing in the magnetic moment. It affects the stable state of the NS structure and thus triggers its transition to another stable state. The magnetic field jumps and takes a larger volume in the $\mathrm{N}$ layer. The new state has a smaller diamagnetic moment which is interpreted experimentally as a «paramagnetic» additive to current.

The numerical renormalized density of states of the NS structure is shown in Fig. 4 as a function of flux at the energy equal to the Fermi energy. It is seen that the period of the density of states jumps decreases as the critical angle $\alpha_{c}$ increases. Finally, at $\alpha_{c}=\pi / 2$ the jumps vanish and the density of states is described by a smooth curve.

The authors of Ref. 13 assumed $\alpha_{c}=\pi / 2$ at which the jumps of the density of states were smoothed. This led the

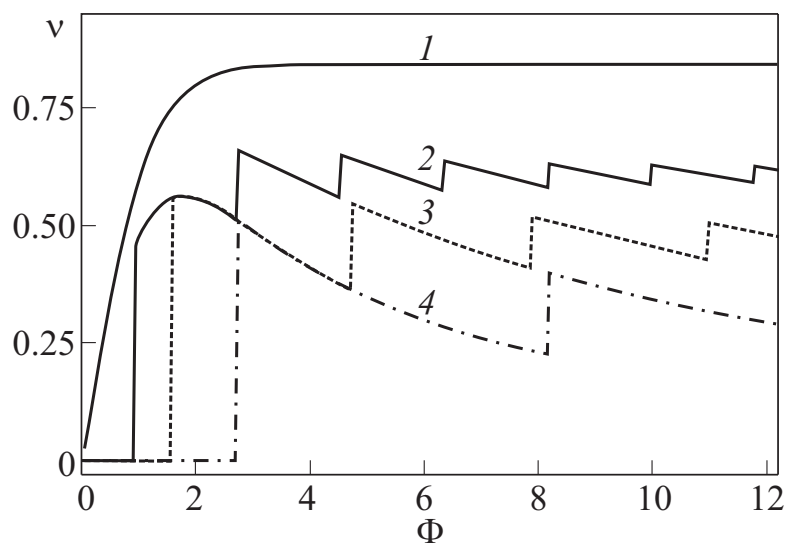

Fig. 4. The density of states near $E=0$ as a function of the flux $\Phi$ at various $\alpha_{c}: \pi / 2(1) ; \pi / 3(2) ; \pi / 4(3) ; \pi / 6(4)$. 


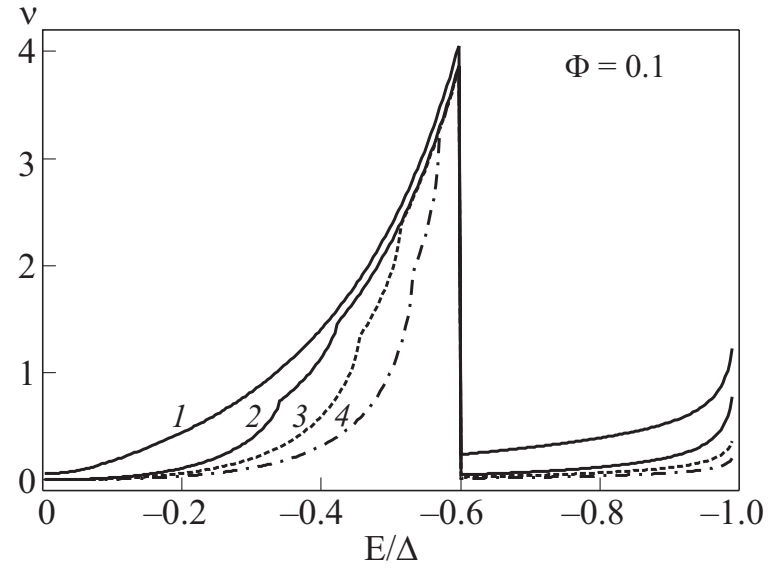

Fig. 5. The density of states as a function of energy at various $\alpha_{c}: \pi / 2(1) ; \pi / 3(2) ; \pi / 4(3) ; \pi / 6(4)$.

authors to a wrong conclusion about the absence of a «paramagnetic» additive to the current of the NS structure.

The dependences of the density of states on energy at different fixed values of the flux $\Phi=0.1$ and 2 are illustrated in Figs. 5 and 6, respectively. It is seen that all the features $v(\varepsilon, \Phi)$ become smoothed at $\alpha_{c}=\pi / 2$.

Detailed information about the isothermal reentrant effect in a NS structure is to appear in another publication.

The authors thank A.N. Omelyanchouk for discussions.

1. P. Visani, A.C. Mota, and A. Pollini, Phys. Rev. Lett. 65, 1514 (1990).

2. A.C. Mota, P. Visani, A. Pollini, and K. Aupke, Physica B197, 95 (1994).

3. F.B. Müller-Allinger and A.C. Mota, Phys. Rev. B62, 6120 (2000).

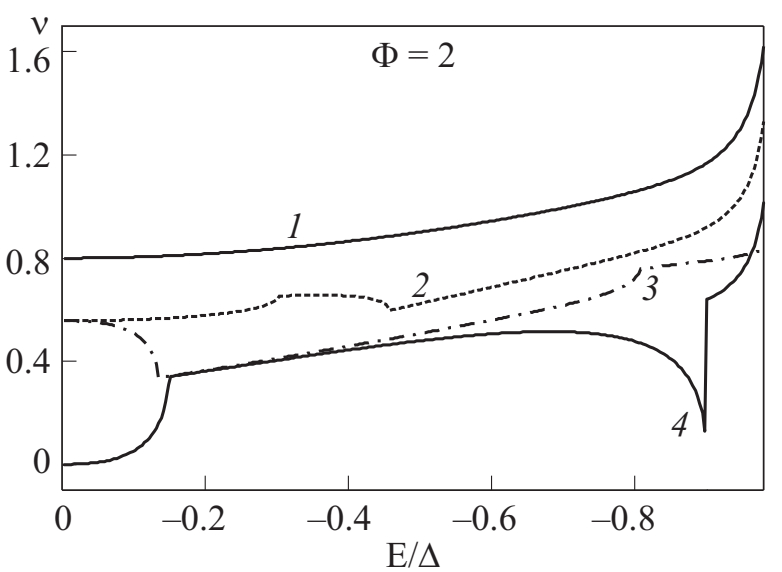

Fig. 6. The density of states as a function of energy at various $\alpha_{c}: \pi / 2(1) ; \pi / 3(2) ; \pi / 4(3) ; \pi / 6(4)$.

4. G.A. Gogadze, Fiz. Nizk. Temp. 31, 120 (2005) [Low Temp. Phys. 31, 94 (2005)].

5. G.A. Gogadze, Fiz. Nizk. Temp. 32, 716 (2006) [Low Temp. Phys. 32, 546 (2006)].

6. G.A. Gogadze, Fiz. Nizk. Temp. 34, 225 (2008) [Low Temp. Phys. 34, 173 (2008)].

7. A.F. Andreev, Zh. Eksp. Teor. Fiz. 46, 1823 (1964) [Sov. Phys. JETP 9, 1228 (1964)].

8. Y. Aharonov and D. Bohm, Phys. Rev. 115, 485 (1959).

9. J.B. Keller and S.I. Rubinow, Ann. Phys. (N.Y.) 9, 24 (1960).

10. G.A. Gogadze, R.I. Shekhter, and M. Jonson, Fiz. Nizk. Temp. 27, 1237 (2001) [Low Temp. Phys. 27, 913 (2001)].

11. A.D. Zaikin, Solid State Commun. 41, 533 (1982).

12. G. Eilenberger, Z. Phys. 214, 195 (1968).

13. A.V. Galaktionov and A.D. Zaikin, Phys. Rev. B67, 184518 (2003). 